\title{
Anti-VEGF treatment patterns and associated health care costs in Switzerland: findings using real-world claims data
}

This article was published in the following Dove Press journal:

Risk Management and Healthcare Policy

2I April 2015

Number of times this article has been viewed

\author{
Oliver Reich' \\ Lucas M Bachmann ${ }^{2}$ \\ Livia Faes ${ }^{3}$ \\ Sophie C Böhni ${ }^{3}$ \\ Mario Bittner ${ }^{3}$ \\ Jeremy P Howell ${ }^{3}$ \\ Michael A Thiel ${ }^{3}$ \\ Roland Rapold' \\ Martin K Schmid ${ }^{3}$ \\ 'Department of Health Sciences, \\ Helsana Group, Dübendorf, \\ 2Medignition Inc, Research \\ Consultants, Zurich, ${ }^{3}$ Eye Clinic, \\ Cantonal Hospital of Lucerne, \\ Lucerne, Switzerland
}

Correspondence: Oliver Reich Head of the Department of Health Sciences, Helsana Group, Zürichstrasse 130, 8600 Dübendorf, Switzerland Email oliver.reich@helsana.ch
Background: Little is known about the patterns of actual health care delivery of anti-vascular endothelial growth factor (VEGF) treatment in patients with age-related macular degeneration, diabetic retinopathy, and retinal vein occlusion in Switzerland. The purpose of this study was to describe these treatment patterns, specifically comparing the numbers of anti-VEGF injections and associated expenditures between patients treated with ranibizumab and those treated with aflibercept in Switzerland using claims data.

Methods: We identified our study patients retrospectively using the Helsana claims database, which includes data on approximately 1.2 million subjects with basic health insurance. Patients qualified for inclusion if ranibizumab or aflibercept had been initiated between December 1 , 2012 (when aflibercept was approved by the Federal Office of Public Health) and November 30, 2013. Within this set, patients with at least 12 months of continuous insurance enrolment in the previous year were considered. In univariate analyses, we examined the distribution of demographic data and patient characteristics between those receiving ranibizumab and those receiving aflibercept. Numbers of injections and associated health care expenditures observed during the 6-month follow-up period after incident treatment were the two outcomes considered. In multivariate regression analyses, controlling for possible confounding factors, we compared differences in these two outcomes between patients treated with ranibizumab and those treated with aflibercept.

Results: Of 3,260 patients who were on anti-VEGF treatment for an ophthalmological indication between December 1, 2012 and November 30, 2013, 1,150 qualified for inclusion. Age, geographic region, and number of physician visits in the previous year were significant factors in the number of injections given during the 6-month follow-up period. Frequency of injections and associated health care expenditures were similar between the groups when correcting for differences in patient characteristics.

Conclusion: Contrary to the recommendations regarding frequency of injections and the results of clinical studies, aflibercept and ranibizumab are used in a similar fashion in Switzerland, resulting in similar total health care expenditures for both these anti-VEGF agents.

Keywords: ranibizumab, aflibercept, health care costs, Switzerland

\section{Introduction}

In Switzerland, two anti-vascular endothelial growth factor (VEGF) medications, ie, ranibizumab (Lucentis ${ }^{\circledR}$ ) and aflibercept $\left(\right.$ Eylea $^{\circledR}$ ), are currently licensed to treat wet age-related macular degeneration (AMD) and retinal vein occlusion (only central vein occlusions for aflibercept), and ranibizumab is also licensed for the treatment of diabetic macular edema. ${ }^{1-3}$ The recombinant fusion protein aflibercept was licensed 
in October 2012 for treatment of neovascular AMD in Switzerland after the VIEW study showed its comparability with ranibizumab with regard to efficacy and safety. ${ }^{2,4}$ In contrast with ranibizumab, which only binds to VEGF-A, ,3,5 aflibercept also binds to VEGF-B and placental growth factor, two additional factors associated with neovascularization. ${ }^{7,8}$ A mathematical model revealing stronger binding affinity of aflibercept to VEGF-165 when compared with ranibizumab suggested that treatment intervals can be extended due to the longer duration of action. ${ }^{9}$ The package insert recommends administration of ranibizumab once monthly after three initial monthly doses. For aflibercept, it is advised to administer the product once a month for the first 3 months followed by once every 2 months. However, little is known about the actual patterns of delivery of anti-VEGF treatment for patients in Switzerland. The "real-world" use of anti-VEGF involving less frequent injections (potentially resulting in less health care expenditure) for aflibercept in comparison with ranibizumab may be different from what is stipulated in the package inserts.

In 2013, Johnston et al published the results of a retrospective analysis of first-line anti-VEGF treatment patterns in AMD based on patient prescription drug claims in the USA. ${ }^{10}$ They compared numbers of injections and associated health care expenditures between patients receiving ranibizumab or aflibercept for 6 or 12 months and found no differences between the two drugs. Whether these findings from the USA apply equally to Swiss circumstances, which has a different population and health care delivery system, is unknown. Therefore, the aim of this study was to describe treatment patterns, specifically comparing the numbers of anti-VEGF injections and associated expenditures, in patients treated with ranibizumab or aflibercept in Switzerland.

\section{Materials and methods}

\section{Swiss health insurance}

Details about the Swiss health care system have been described in detail elsewhere. ${ }^{11}$ In brief, Switzerland has a population of approximately 8 million and is organized as a federal parliamentary republic with 26 cantons. The Swiss health care system has mandatory coverage and is consumer-driven. Consumers pay health care costs through insurance premiums and out-of-pocket expenditures. In addition, the coverage has a mandatory cost-sharing scheme consisting of copayments and deductibles for all residents. Copayments are a charge of $10 \%$ of the annual health care costs that every insured person has to pay.

\section{Data source and patient identification}

Helsana is the leading health insurance group in Switzerland. Retrospectively, we identified patients using the Helsana claims database, which includes approximately 1.2 million subjects with basic health insurance and provides an approximate representativeness of the general population (representing $15 \%$ of 8.04 million inhabitants). Patients qualified for inclusion if treatment with ranibizumab or aflibercept had been initiated between December 1, 2012 (the Federal Office of Public Health approval date for aflibercept) and November 30, 2013. All prescription drug items are coded and assigned to a specific pharmaceutical code in our database. On this basis, evidence of intravitreal injections with aflibercept or ranibizumab was acquired by using the corresponding pharmaceutical code. Of this set, patients with at least 12 months of continuous insurance enrolment in the previous year were considered. In order to focus our analysis on patients who had initiated first-line intravitreally injected anti-VEGF treatment, individuals who received any ranibizumab or aflibercept prescription medication in the year before their individual incident treatment date were excluded from the sample. We also excluded patients in whom both eyes were treated. For the purposes of this analysis, we focused on patients with a follow-up period of at least 6 months and anti-VEGF prescriptions from ambulatory care only.

The characteristics of our sample comprised sex, age, health plan coverage (deductible class, ie, standard deductible of 300, 500, 1,000, 1,500, 2,000, or 2,500 Swiss francs (CHF), managed care option, and availability of supplementary private hospital insurance), region of domicile and presence of chronic condition using pharmaceutical cost groups. If information on medical diagnosis is missing in the available data set, pharmaceutical cost groups are established individual markers for selected 22 chronic conditions. ${ }^{12}$ Therefore, the chronic conditions included in this study were diabetes mellitus, cardiovascular disease, glaucoma, hyperacidity-related disorders, bone diseases (osteoporosis), cancer, dementia, epilepsy, gout/hyperuricemia, human immunodeficiency virus infection, hyperlipidemia, intestinal inflammatory diseases, iron deficiency anemia, migraine, pain, Parkinson's disease, psychological disorders, psychoses, respiratory illness, rheumatological conditions, thyroid disorders, and tuberculosis. Our data set also included information on health service utilization, number of anti-VEGF injections, prescription of other than the aforementioned anti-VEGF drugs, and their associated costs from outpatient and inpatient health care settings. 


\section{Statistical analysis}

Data were anonymized and prepared for statistical analysis. Continuous variables were described as the mean or median. Dichotomous variables were described with percentages. In univariate analyses, we examined the distribution of demographic data and patient characteristics between those receiving ranibizumab and those receiving aflibercept. Differences between groups were statistically tested with parametric or nonparametric tests as appropriate. Numbers of injections and associated health care expenditures observed during the 6-month follow-up period after incident treatment were the two outcomes considered. In multivariate regression analyses, controlling for possible confounding factors, we compared differences in the two outcomes (number of injections and associated health care expenditures) between patients receiving ranibizumab and those receiving aflibercept. A $P$-value of less than five percent was considered to be statistically significant. Analyses were performed using $\mathrm{R}$ version 3.1.0 (R Foundation for Statistical Computing, Vienna, Austria). ${ }^{13}$

\section{Ethics}

In compliance with national ethical and legal regulations, all data were anonymized and deidentified prior to data analysis to protect the privacy of patients, physicians, and hospitals. Because the data were retrospective, pre-existing, and deidentified, this study was exempted from ethics committee approval.

\section{Results}

Of 3,260 patients on anti-VEGF treatment for an ophthalmological indication between December 1, 2012 and November 30, 2013, 1,150 qualified for inclusion in this study. The patient flow and reasons for exclusion are outlined in Figure 1.

\section{Patient characteristics and health care utilization}

The demographic distribution between patients treated with aflibercept and those treated with ranibizumab are summarized in Tables 1 and 2. Overall, there was a higher percentage of women $(67.5 \%$ versus $60.6 \%)$ in the aflibercept sample. The mean age was 80.0 (median 81.0 ) years for aflibercept patients and 76.4 (median 79.0) years for ranibizumab patients. There were differences in the shape of the distribution of age between the treatment groups. Ranibizumab patients showed wider variability (standard deviation [SD]

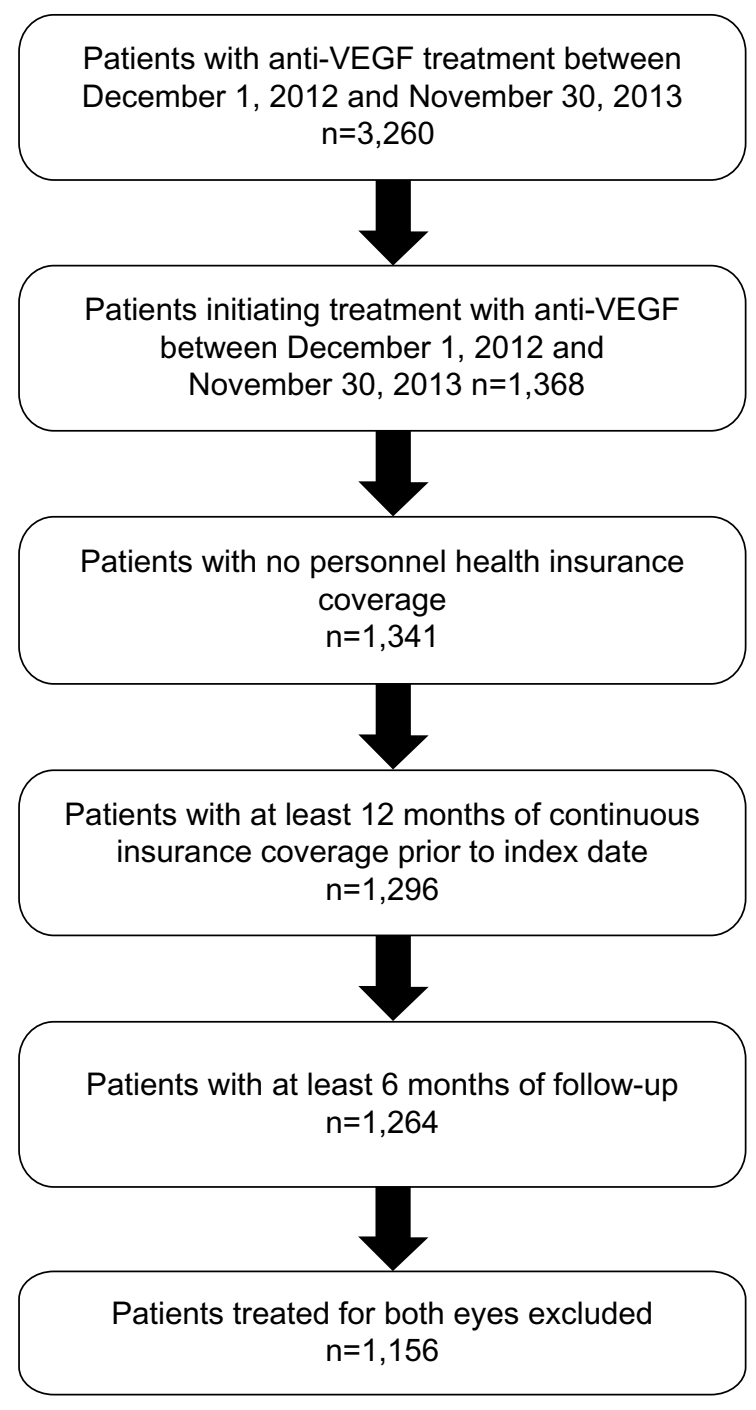

Figure I Patient flow.

Abbreviation: VEGF, vascular endothelial growth factor.

11.3, interquartile range 13) than aflibercept patients (SD 8.3, interquartile range 10). When examining insurance coverage (deductible class, supplementary hospital insurance, managed care model), aflibercept patients generally did not differ substantially from ranibizumab patients. There were large differences between aflibercept and ranibizumab prescriptions across the different regions of Switzerland. The proportion of patients treated with aflibercept was highest in the region of Middle land (28.5\%). The highest proportion of patients treated with ranibizumab was in the central region of Switzerland $(87.4 \%)$. The unadjusted mean health care expenditure on intravitreal anti-VEGF injections during the first 6 months after the index date was CHF 13,856 in the ranibizumab patients and CHF 13,484 in the aflibercept patients. The mean number of chronic conditions was 3.8 (SD 2.1) among 
Table I Distribution of demographic data

\begin{tabular}{|c|c|c|c|c|}
\hline Variable & Total & Ranibizumab & Aflibercept & $P$-value \\
\hline $\mathrm{n}$ & 1,150 & 895 & 255 & \\
\hline Female (\%) & 62.1 & 60.6 & 67.5 & 1.000 \\
\hline Age (mean, median) & $77.2(79.0)^{*}$ & $76.4(79.0)$ & $80.0(81.0)$ & $<0.001$ \\
\hline Standard deviation (IQR) & $10.8(\mid 3.0)$ & II.3 (I3.0) & $8.3(10.0)$ & \\
\hline Age group (\%) & & & & 1.000 \\
\hline $19-25$ & 0.2 & 0.2 & 0.0 & \\
\hline $26-30$ & 0.2 & 0.2 & 0.0 & \\
\hline $31-35$ & 0.3 & 0.4 & 0.0 & \\
\hline $36-40$ & 0.3 & 0.3 & 0.0 & \\
\hline $4 I-45$ & 0.6 & 0.8 & 0.0 & \\
\hline $46-50$ & 0.8 & 1.0 & 0.0 & \\
\hline $5 \mathrm{I}-55$ & 1.6 & 1.7 & 1.2 & \\
\hline $56-60$ & 3.3 & 3.9 & 1.2 & \\
\hline $6 I-65$ & 6.2 & 6.8 & 3.9 & \\
\hline $66-70$ & 8.5 & 9.2 & 6.3 & \\
\hline $71-75$ & 12.4 & 13.4 & 9.0 & \\
\hline $76-80$ & 21.8 & 20.8 & 25.5 & \\
\hline $81-85$ & 22.6 & 21.5 & 26.7 & \\
\hline $86-90$ & 15.5 & 14.5 & 18.8 & \\
\hline $91-$ & 5.7 & 5.3 & 7.5 & \\
\hline Deductible class & 69.7 & 68.8 & 72.5 & 1.000 \\
\hline \multicolumn{5}{|l|}{ CHF $300(\%)$} \\
\hline Deductible class & 30.3 & 31.2 & 27.5 & 1.000 \\
\hline \multicolumn{5}{|l|}{$>$ CHF $300(\%)$} \\
\hline Supplementary insurance coverage hospital (\%) & 27.9 & 28.1 & 27.4 & 1.000 \\
\hline Managed care insurance $(\%)$ & 34.2 & 33.9 & 35.3 & 1.000 \\
\hline Geographic region (\%) & & & & 1.000 \\
\hline Geneva Lake & 100 & 76.4 & 23.6 & \\
\hline Middle land & 100 & 71.5 & 28.5 & \\
\hline Northwest & 100 & 85.3 & 14.7 & \\
\hline East & 100 & 77.5 & 22.5 & \\
\hline Ticino & 100 & 75.3 & 24.7 & \\
\hline Central & 100 & 87.4 & 12.6 & \\
\hline Zurich & 100 & 77.1 & 22.9 & \\
\hline
\end{tabular}

Notes: *Significance level. The Kruskal-Wallis test was used to check for significant differences in age and the chi-square test for differences in categorical variables. CHF denotes Swiss Francs.

Abbreviation: IQR, interquartile range.

ranibizumab patients and significantly higher compared with aflibercept patients (mean 3.5, SD 2.0). Further, patients on ranibizumab had significantly higher mean numbers of drug prescriptions (13.3 versus 12.5) and physicians' visits (11.1 versus 10.3). The mean unadjusted number of intravitreal anti-VEGF injections over the first 6 months after the index date was 3.91 (SD 1.66) in the aflibercept patients and 3.86 (SD 1.78) in the ranibizumab patients.

\section{Numbers of injections}

\section{and health care expenditures}

Multivariate regression adjusting for patient demographics and potential confounders determined that the number of injections was not significantly different between aflibercept patients and ranibizumab patients. Factors that were significantly associated with receiving more intravitreal anti-VEGF injections included older age and living in the East, Central, and Zurich regions. Health care expenditures were similar between the two treatment groups when correcting for differences in patient characteristics operationalized with the variable total health care costs in the previous year. The results are summarized in Tables 3 and 4.

\section{Discussion}

Despite differences in the prescribing information regarding recommendations for the frequency of injections, both antiVEGF agents were used nearly equally. Neither numbers of anti-VEGF injections nor the associated health care costs differed significantly between patients initially treated with aflibercept and those initially treated with ranibizumab. We found considerable differences in treatment patterns across the different regions of Switzerland. We do not have a good explanation for this. Arguably, treatment strategies are made under the assumption of equivalence, and treatment choice 
Table 2 Distribution of patient characteristics, health care utilization, and costs

\begin{tabular}{|c|c|c|c|c|}
\hline Variable & Total & Ranibizumab & Aflibercept & $P$-value \\
\hline Total costs in CHF, mean (median) & I3,773 ( 1 I,687) & $13,856(\mid 1,634)$ & 13,484 (I I,842) & 0.961 \\
\hline Standard deviation (IQR) & $9,57 \mid(7,032)$ & $9,967(7,156)$ & $8,043(6,428)$ & \\
\hline Outpatient costs in CHF, mean (median) & I I,777 (I0,948) & II,9I2 (I0,95I) & II,305 (10,937) & 0.716 \\
\hline Standard deviation (IQR) & $6,246(5,734)$ & $6,684(6,064)$ & $4,356(4,813)$ & \\
\hline Medication costs in CHF, mean (median) & $5,164(4,833)$ & $5,192(4,869)$ & $5,066(4,684)$ & 0.997 \\
\hline Standard deviation (IQR) & $2,684(2,820)$ & $2,842(2,888)$ & $2,035(2,375)$ & \\
\hline Anti-VEGF medication costs in CHF, mean (median) & $4,114(4,268)$ & $4,102(4,268)$ & $4,155(4,268)$ & 0.568 \\
\hline Standard deviation (IQR) & $\mathrm{I}, 873(2,134)$ & $1,900(2,134)$ & $\mathrm{I}, 778(2,134)$ & \\
\hline Inpatient costs in CHF, mean (median) & $\mathrm{I}, 996(0)$ & $\mathrm{I}, 944(0)$ & $2,179(0)$ & 0.820 \\
\hline Standard deviation (IQR) & $6,832(0)$ & $6,870(0)$ & $6,707(0)$ & \\
\hline Hospitalizations, mean (median) & $0.20(0)$ & $0.20(0)$ & $0.17(0)$ & 0.383 \\
\hline Standard deviation (IQR) & $0.53(0)$ & $0.55(0)$ & $0.49(0)$ & \\
\hline None (\%) & 85.0 & 84.5 & 86.7 & 1.000 \\
\hline One (\%) & 11.9 & 12.3 & 10.6 & \\
\hline Two (\%) & 2.1 & 2.0 & 2.4 & \\
\hline At least three (\%) & 1.0 & 1.2 & 0.4 & \\
\hline Physician visits, mean (median) & $10.9(10.0)^{*}$ & II.I (I0.0) & $10.3(9.0)$ & 0.129 \\
\hline Standard deviation (IQR) & $7.8(10.0)$ & $7.9(10.0)$ & $7.3(8.0)$ & \\
\hline $0(\%)$ & 3.7 & 3.5 & 4.7 & 1.000 \\
\hline $1-4(\%)$ & 16.5 & 17.0 & 14.9 & \\
\hline $5-10(\%)$ & 32.3 & 30.3 & 39.2 & \\
\hline I I-20 (\%) & 38.3 & 39.3 & 34.5 & \\
\hline$\geq 21(\%)$ & 9.2 & 9.9 & 6.7 & \\
\hline Outpatient hospital visits, mean (median) & $5.8(5.0)$ & $5.9(4.0)$ & $5.7(5.0)$ & 0.748 \\
\hline Standard deviation (IQR) & $7.7(7.0)$ & $8.1(7.0)$ & $5.7(9.0)$ & \\
\hline $0(\%)$ & 23.0 & 22.0 & 26.3 & 1.000 \\
\hline $\mathrm{I}-4(\%)$ & 27.0 & 28.7 & 20.8 & \\
\hline $5-10(\%)$ & 35.2 & 35.4 & 34.5 & \\
\hline II-20 (\%) & 12.3 & 11.3 & I6.I & \\
\hline$\geq 21(\%)$ & 2.5 & 2.6 & 2.4 & \\
\hline Number of chronic diseases, mean (median) & $3.7(4.0)^{*}$ & $3.8(4.0)$ & $3.5(3.0)$ & 0.037 \\
\hline Standard deviation (IQR) & $2.0(3.0)$ & $2.1(3.0)$ & $2.0(3.0)$ & \\
\hline $0(\%)$ & 5.5 & 5.6 & 5.1 & \\
\hline I (\%) & 9.6 & 8.9 & II.8 & 1.000 \\
\hline $2(\%)$ & 14.3 & 14.0 & 15.7 & \\
\hline $3(\%)$ & 16.6 & 15.9 & 19.2 & \\
\hline 4-6 (\%) & 44.5 & 45.8 & 40.0 & \\
\hline$\geq 7(\%)$ & 9.5 & 9.8 & 8.2 & \\
\hline Number of different drugs (ATC codes), mean (median) & I3.I (12.0)* & $13.3(13.0)$ & $12.5(\mid \mathrm{I} .0)$ & 0.040 \\
\hline Standard deviation (IQR) & $6.7(9.0)$ & $6.7(9.0)$ & $6.7(9.0)$ & \\
\hline $0(\%)$ & 0.1 & 0.1 & 0.0 & 1.000 \\
\hline $1-4(\%)$ & 7.7 & 7.5 & 8.6 & \\
\hline $5-10(\%)$ & 31.7 & 30.1 & 37.6 & \\
\hline II-20 (\%) & 47.7 & 49.1 & 42.7 & \\
\hline$\geq 21(\%)$ & 12.8 & 13.3 & II.0 & \\
\hline Anti-VEGF injections, mean (median) & $3.88(4.0)$ & $3.86(4.0)$ & $3.91(4.0)$ & 0.521 \\
\hline Standard deviation (IQR) & $\mathrm{I} .76(2.0)$ & $\mathrm{I} .78(2.0)$ & $1.66(2.0)$ & \\
\hline I (\%) & 8.6 & 8.9 & 7.5 & 0.570 \\
\hline $2(\%)$ & 9.9 & 10.1 & 9.4 & \\
\hline $3(\%)$ & 28.6 & 29.1 & 27.1 & \\
\hline $4(\%)$ & 18.5 & 17.9 & 20.8 & \\
\hline 5-6 (\%) & 29.7 & 29.6 & 30.2 & \\
\hline 7-9 (\%) & 3.5 & 3.1 & 4.7 & \\
\hline$\geq 10(\%)$ & I.I & 1.3 & 0.4 & \\
\hline
\end{tabular}

Notes: CHF denotes Swiss francs. *Significance level. The Kruskal-Wallis test was used to check for significant differences between groups.

Abbreviations: ATC, Anatomical Therapeutic Classification; IQR, interquartile range; VEGF, vascular endothelial growth factor.

is made on the basis of physicians' preference for one of the two drugs. However, patients receiving aflibercept were older but had less comorbidity, indicating possible tacit risk selection.
In this analysis, patient age was associated with frequency of injections. Our opportunity to study this phenomenon in detail is limited, since clinical data, allowing exploration of illness-associated reasons, were unavailable. We cannot rule 
Table 3 Multivariable Poisson regression of number of anti-VEGF injections over the first 6 months after the index date

\begin{tabular}{|c|c|c|c|c|}
\hline Variables & Count ratio & $95 \% \mathrm{Cl}$ & $P$-value & Level \\
\hline (Intercept) & 0.90 & $(0.22-3.62)$ & 0.884 & \\
\hline Anti-VEGF medication (aflibercept) & 1.02 & $(0.95-1.10)$ & 0.525 & \\
\hline Sex (female) & 1.04 & $(0.97-1.10)$ & 0.265 & \\
\hline \multicolumn{5}{|l|}{ Age group, years (19-25) } \\
\hline $26-30$ & 1.00 & $(0.14-7.10)$ & 1.00 & \\
\hline $3 I-35$ & 2.41 & $(0.52-11.16)$ & 0.262 & \\
\hline $36-40$ & 3.12 & $(0.66-|4.7|)$ & 0.151 & \\
\hline $4 I-45$ & 3.92 & $(0.93-16.53)$ & 0.063 & * \\
\hline $46-50$ & 4.58 & $(1.10-18.98)$ & 0.036 & $* *$ \\
\hline $5 \mathrm{I}-55$ & 4.08 & $(1.00-16.69)$ & 0.050 & * \\
\hline $56-60$ & 4.51 & $(1.12-18.20)$ & 0.035 & $* *$ \\
\hline $61-65$ & 4.54 & $(1.13-18.25)$ & 0.033 & $* *$ \\
\hline $66-70$ & 4.54 & $(1.13-18.25)$ & 0.033 & $* *$ \\
\hline $7 I-75$ & 4.31 & $(1.07-17.28)$ & 0.039 & $* *$ \\
\hline $76-80$ & 4.18 & $(1.04-16.75)$ & 0.044 & $* *$ \\
\hline $8 I-85$ & 4.21 & $(1.05-16.89)$ & 0.042 & $* *$ \\
\hline $86-90$ & 4.00 & $(1.00-16.05)$ & 0.050 & $*$ \\
\hline$\geq 91$ & 3.47 & $(0.86-13.97)$ & 0.080 & $*$ \\
\hline \multicolumn{5}{|l|}{ Region (Geneva Lake) } \\
\hline Middle land & 0.97 & $(0.87-1.08)$ & 0.585 & \\
\hline Northwest & 0.97 & $(0.86-1.08)$ & $0.54 I$ & \\
\hline East & 1.06 & $(0.94-1.20)$ & 0.350 & \\
\hline South (Tessin) & 0.81 & $(0.70-0.93)$ & 0.003 & $* * *$ \\
\hline Central & 1.08 & $(0.95-1.24)$ & $0.24 I$ & \\
\hline Zurich & 1.06 & $(0.96-1.17)$ & 0.269 & \\
\hline
\end{tabular}

Notes: $* p<0.10, * * p<0.05$, **** $p<0.01$.

Abbreviations: $\mathrm{Cl}$, confidence interval; VEGF, vascular endothelial growth factor.

out elderly patients receiving less intensive treatment for organizational reasons. Following a pro re nata treatment scheme, for instance, requires substantial support of (para-) medical personnel offering transport, patient companionship, and other assistance. The relationship between injection frequency and geographic region could be explained along similar lines. Compared with an urban setting, these organizational aspects may play an even more important role in rural areas such as the South of Switzerland. However, unfortunately, these specific aspects of health care delivery have not yet been studied carefully and remain an area for further research.

Moreover, differences in indications per treatment due to differences in drug labeling might be another explanation for the demographic differences found. However, while patients with diabetes might have a higher number of comorbidities, they also tend to be younger than patients with AMD. In view of the fact that approximately $90 \%$ of patients receiving anti-VEGF medication suffer from AMD, but less than $10 \%$ have diabetic retinal edema, ${ }^{14}$ we believe that these effects do not fully explain the differences between treatment groups.

We are unaware of any study examining clinical outcomes and costs of ophthalmological anti-VEGF management within a health service research paradigm in a broader context in Switzerland. Our results essentially corroborate the findings of Johnston et al from the USA. ${ }^{10}$ They used administrative claims data and compared numbers of injections and health care expenditures between patients receiving ranibizumab or aflibercept for 6 or 12 months and found no differences in these parameters between the two drugs.

To our knowledge, this is the first study identifying treatment patterns, specifically comparing numbers of anti-VEGF injections and associated expenditures between patients treated with ranibizumab and those treated with aflibercept in Switzerland. Due to lack of clinical data, we were unable to make a distinction between the underlying diseases. Therefore, it was impossible to assess variability in costs between AMD, diabetic macular edema, and retina vein occlusion. Finally, although representing a large proportion of Swiss citizens, we cannot rule out that patient selection prevented us from being able to extrapolate our results to the whole country. Nevertheless, we believe that our results are valid enough to inform a discussion about anti-VEGF treatment patterns in Switzerland. The concordance with findings in other countries such as the USA is encouraging. 
Table 4 Multivariable linear logistic regression of health care expenditures over the first 6 months after the index date

\begin{tabular}{|c|c|c|c|c|}
\hline Variable & Estimate & $95 \% \mathrm{Cl}$ & $P$-value & Level \\
\hline (Intercept) & $3,555.35$ & $(1,788.68-7,066.95)$ & $<0.001$ & $* * *$ \\
\hline Anti-VEGF medication (aflibercept) & 1.02 & $(0.95-1.09)$ & 0.608 & \\
\hline Sex (female) & 1.00 & $(0.94-1.06)$ & 0.963 & \\
\hline \multicolumn{5}{|l|}{ Age, years } \\
\hline $26-30$ & 0.68 & $(0.26-1.77)$ & 0.432 & \\
\hline $31-35$ & 1.72 & $(0.75-3.93)$ & 0.199 & \\
\hline $36-40$ & 1.92 & $(0.80-4.59)$ & 0.142 & \\
\hline $4 I-45$ & 2.00 & $(0.93-4.31)$ & 0.078 & $*$ \\
\hline $46-50$ & 1.98 & $(0.94-4.19)$ & 0.073 & $*$ \\
\hline $5 I-55$ & 2.43 & $(1.19-4.97)$ & 0.015 & $* *$ \\
\hline $56-60$ & 2.41 & $(1.21-4.84)$ & 0.013 & $* *$ \\
\hline $6 I-65$ & 2.37 & $(I .19-4.7 I)$ & 0.014 & $* *$ \\
\hline $66-70$ & 2.19 & $(1.11-4.34)$ & 0.025 & $* *$ \\
\hline $7 I-75$ & 2.20 & $(1.11-4.34)$ & 0.024 & ** \\
\hline $76-80$ & 2.26 & $(1.15-4.46)$ & 0.019 & $* *$ \\
\hline $81-85$ & 2.45 & $(1.24-4.84)$ & 0.010 & $* * *$ \\
\hline $86-90$ & 2.35 & $(1.19-4.63)$ & 0.014 & $* *$ \\
\hline$\geq 91$ & 2.32 & $(1.17-4.61)$ & 0.017 & $* *$ \\
\hline \multicolumn{5}{|l|}{ Total health care costs ${ }^{\mathrm{a}}$ previous year } \\
\hline Class I & 1.15 & $(I .0 I-\mid .3 I)$ & 0.035 & $* *$ \\
\hline Class 2 & 1.26 & $(1.10-1.44)$ & 0.001 & $* * *$ \\
\hline Class 3 & 1.37 & $(1.20-1.57)$ & $<0.001$ & $* * *$ \\
\hline Class 4 & 1.36 & $(1.19-1.55)$ & $<0.001$ & $* * *$ \\
\hline Class 5 & 1.43 & $(1.26-1.64)$ & $<0.001$ & $* * *$ \\
\hline Class 6 & 1.42 & $(1.24-1.62)$ & $<0.001$ & $* * *$ \\
\hline Class 7 & 1.54 & $(1.35-1.76)$ & $<0.001$ & **** \\
\hline Class 8 & 1.69 & $(1.48-1.93)$ & $<0.001$ & $* * *$ \\
\hline Class 9 & 2.66 & $(2.32-3.05)$ & $<0.001$ & $* * *$ \\
\hline
\end{tabular}

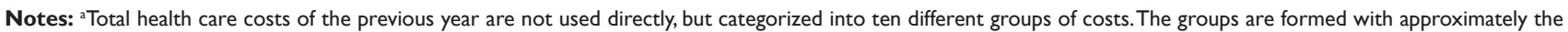
same number of patients per group (10\% per group), whereas the first group with the lowest costs in the previous year is the according reference value. $* P<0.10,{ }^{* * P}<0.05$, $* * * P<0.01$.

Abbreviations: $\mathrm{Cl}$, confidence interval; VEGF, vascular endothelial growth factor.

\section{Conclusion}

Contrary to treatment recommendations regarding the frequency of injections and the results from clinical studies, both anti-VEGF agents are used in a similar fashion, resulting in similar total health care expenditures for aflibercept and ranibizumab.

\section{Disclosure}

Financial support for this study was provided by Novartis Pharma Schweiz AG, Switzerland. The sponsor had no role in study design; collection, analysis, and interpretation of data; writing of the paper; or in the decision to submit the paper for publication. OR and RR received funding from Novartis Pharma Schweiz AG, Switzerland. MKS, MAT, LF, SCB, $\mathrm{MB}, \mathrm{JPH}$, and LMB have no conflicts of interest to report.

\section{References}

1. Brown DM, Michels M, Kaiser PK, Heier JS, Sy JP, Ianchulev T; ANCHOR Study Group. Ranibizumab versus verteporfin for neovascular age-related macular degeneration. $N$ Engl J Med. 2006;355(14): $1432-1444$.
2. Heier JS, Brown DM, Chong V, et al. Intravitreal aflibercept (VEGF trap-eye) in wet age-related macular degeneration. Ophthalmology. 2012;119(12):2537-2548.

3. Rosenfeld PJ, Brown DM, Heier JS, et al. Ranibizumab for neovascular age-related macular degeneration. $N$ Engl J Med. 2006;355(14): $1419-1431$.

4. Schmidt-Erfurth U, Kaiser PK, Korobelnik JF, et al. Intravitreal aflibercept injection for neovascular age-related macular degeneration: ninetysix-week results of the VIEW studies. Ophthalmology. 2014;121(1): 193-201.

5. Ferrara N, Davis-Smyth T. The biology of vascular endothelial growth factor. Endocr Rev. 1997;18(1):4-25.

6. Grisanti S, Tatar O. The role of vascular endothelial growth factor and other endogenous interplayers in age-related macular degeneration. Prog Retin Eye Res. 2008;27(4):372-390.

7. Papadopoulos N, Martin J, Ruan Q, et al. Binding and neutralization of vascular endothelial growth factor (VEGF) and related ligands by VEGF Trap, ranibizumab and bevacizumab. Angiogenesis. 2012;15(2): 171-185.

8. Rakic JM, Lambert V, Munaut C, et al. Mice without uPA, tPA, or plasminogen genes are resistant to experimental choroidal neovascularization. Invest Ophthalmol Vis Sci. 2003;44(4):1732-1739.

9. Stewart MW, Rosenfeld PJ. Predicted biological activity of intravitreal VEGF Trap. Br J Ophthalmol. 2008;92(5):667-668.

10. Johnston SS, Wilson K, Huang A, Smith D, Varker H, Turpcu A. Retrospective analysis of first-line anti-vascular endothelial growth factor treatment patterns in wet age-related macular degeneration. $A d v$ Ther. 2013;30(12):1111-1127. 
11. Herzlinger RE, Parsa-Parsi R. Consumer-driven health care: lessons from Switzerland. JAMA. 2004;292(10):1213-1220.

12. Huber CA, Szucs TD, Rapold R, Reich O. Identifying patients with chronic conditions using pharmacy data in Switzerland: an updated mapping approach to the classification of medications. BMC Public Health. 2013;13:1030.

13. The R Project for Statistical Computing. R: A language and environment for statistical computing. Vienna, Austria: R Foundation for Statistical Computing; 2008. Available from: http://www.R-project.org. Accessed February 28, 2015.
14. Brand CS. Baseline characteristics of over 10,000 patients enrolled into the LUMINOUS study. Available from: http://www.arvo.org/webs/ am2014/abstract/sessions/335.pdf. Accessed February 28, 2015.

\section{Publish your work in this journal}

Risk Management and Healthcare Policy is an international, peerreviewed, open access journal focusing on all aspects of public health, policy, and preventative measures to promote good health and improve morbidity and mortality in the population. The journal welcomes submitted papers covering original research, basic science, clinical \& epidemio- logical studies, reviews and evaluations, guidelines, expert opinion and commentary, case reports and extended reports. The manuscript management system is completely online and includes a very quick and fair peerreview system, which is all easy to use. Visit http://www.dovepress.com/ testimonials.php to read real quotes from published authors.

Submit your manuscript here: http://www.dovepress.com/risk-management-and-healthcare-policy-journal 